\title{
SPECTRAL GAP ESTIMATES FOR SOME BLOCK MATRICES
}

\author{
IVAN VESELIĆ AND KREŠIMIR VESELIĆ
}

Abstract. We estimate the size of the spectral gap at zero for some Hermitian block matrices. Included are quasi-definite matrices, quasi-semidefinite matrices (the closure of the set of the quasi-definite matrices) and some related block matrices which need not belong to either of these classes. Matrices of such structure arise in quantum models of possibly disordered systems with supersymmetry or graphene like symmetry. Some of the results immediately extend to infinite dimension.

Mathematics subject classification (2010): 15A60, 47A75, 15A22.

Keywords and phrases: spectral gap estimates; block matrices; quais-definite matrices; Stokes matrices; Dirac symmetry; spectral pollution.

\section{REFERENCES}

[1] O. Axelsson, M. Neytcheva, Eigenvalue estimates for preconditioned saddle point matrices, Numerical Linear Algebra 13 (2006) 339-360.

[2] C. S. Ballantine, Products of positive definite matrices, J. Algebra 10 (1968) 74-182.

[3] E. Cancès, V. Ehrlacher, Y. Maday, Periodic Schrödinger operators with local defects and spectral pollution, SIAM Journal on Numerical Analysis, 50 (2012) 3016-3035.

[4] J. Chapman, G. Stolz, Localization for random block operators related to the $X Y$ spin chain, http://arxiv.org/abs/1308.0708.

[5] R. J. Duffin, A minimax theory for overdamped networks, J. Rational Mech. Anal. 4 (1955), $221-$ 233.

[6] J. ERXIONG, Bounds for the smallest singular value of a Jordan block with an application to eigenvalue perturbation, LAA 197 (1994) 697-707.

[7] M. Hladnik, M. Omladič, Spectrum of the product of operators, Proc. Amer. Math. Soc. 102 (1988) 300-302.

[8] Y. HONG, R. A. HoRn, The Jordan canonical form of a product of a Hermitian and a positive semidefinite matrix, LAA 147 (1991) 373-386.

[9] A. George, Kh. Ikramov, A. B. Kucherov, Some properties of symmetric quasi-definite matrices, SIAM J. Matrix Anal. Appl. 21 (2000) 1318-1323.

[10] T. KATO, Perturbation Theory for Linear Operators, Springer 1966.

[11] H. VAN KEMPEN, Variation of the eigenvalues of a special class of Hermitian matrices upon variation of some of its elements, LAA 3 (1970), 263-273.

[12] W. Kirsch, B. Metzger, P. Müller, Random block operators, J. Stat. Phys. 143 (2011) 10351054.

[13] G. A. MeZINCESCU, Internal Lifschitz singularities for one dimensional Schrödinger operators, Comm. Math. Phys. 158 (1993) 315-325.

[14] T. RUSTEN, R. WINTHER, A preconditioned iterative method for saddlepoint problems, SIMAX 13 (1992) 887-904.

[15] M. A. SAUNDERS, Solution of sparse rectangular systems using lsqr and craig, BIT 25 (1995) 588604.

[16] S. Schmitz, Representation theorems for indefinite quadratic forms and applications, PhD thesis University of Mainz 2014.

[17] R. Thaller, The Dirac Equation, Springer 1992. 
[18] R. C. ThOMPSOn, The Eigenvalues of a partitioned Hermitian matrix involving a parameter, LAA 9 (1974) 243-260.

[19] K. VESELIĆ, Spectral perturbation bounds for selfadjoint operators I, Operators and Matrices 2 (2008) 307-340.

[20] K. VeSElić, I. SlapniČAR, Floating point perturbations of Hermitian matrices, Linear Algebra Appl. 195 (1993) 81-116.

[21] M. Winklmeier, The Angular Part of the Dirac Equation in the Kerr-Newman Metric: Estimates for the Eigenvalues, Ph. D. Thesis, 2005. 


\section{A. Some auxiliary computations}

\section{Proof of equation (??)}

Since the matrices of the type (??) appear to be the source of many illustrative examples we here give an explicit formula for their eigenvalues (which come in plus/minus pairs). We put

$$
A=\left[\begin{array}{cc}
a_{+} & a \\
\bar{a} & a_{-}
\end{array}\right], \quad B=\left[\begin{array}{cc}
b_{+} & b \\
\pm \bar{b} & b_{-}
\end{array}\right],
$$

so that in the case of the minus sign in $B$ the diagonal elements $b_{ \pm}$are purely imaginary. A straightforward calculation gives

$$
\left(\lambda^{2}\right)_{1,2}=\frac{s}{2} \pm \sqrt{s^{2}-\left.\left|a_{+} a_{-}-b_{+} b_{-}-\right| a\right|^{2}+\left.|b|^{2}\right|^{2}-\left|a_{+} b_{-}-b_{+} a_{-}-2 \Re \bar{a} b\right|^{2}} .
$$

with

$$
s=\frac{a_{+}^{2}+a_{-}^{2}+\left|b_{+}\right|^{2}+\left|b_{-}\right|^{2}}{2}+|a|^{2}+|b|^{2} .
$$

\section{Proof of equation (??)}

We derive the formula (??). Substituting $x_{j}=A \cos j \alpha+B \sin j \alpha$ in (??) we get

$$
\begin{aligned}
A \cos j \alpha \cos \alpha & +A \sin j \alpha \sin \alpha B \sin j \alpha \cos \alpha-B \cos j \alpha \sin \alpha \\
& +\kappa(A \cos j \alpha+B \sin j \alpha) \\
+A \cos j \alpha \cos \alpha & -A \sin j \alpha \sin \alpha B \sin j \alpha \cos \alpha+B \cos j \alpha \sin \alpha \\
& =0
\end{aligned}
$$

or

$$
(A \cos j \alpha+B \sin j \alpha)(\cos \alpha+\cos \alpha+\kappa)=0
$$

thus implying

$$
\kappa=-2 \cos \alpha
$$

The boundary conditions (??) yield

$$
\begin{aligned}
A & =-A \cos \alpha-B \sin \alpha, \\
A \cos (m+1) \alpha+B \sin (m+1) \alpha & =A \cos m \alpha+B \sin m \alpha,
\end{aligned}
$$

which is a homogeneous linear system

$$
\begin{aligned}
(1+\cos \alpha) A+B \sin \alpha & =0, \\
A(\cos (m+1) \alpha-\cos m \alpha)+B(\sin (m+1) \alpha-\sin m \alpha) & =0,
\end{aligned}
$$

so its determinant must vanish:

$$
(1+\cos \alpha) 2 \cos \frac{(2 m+1) \alpha}{2} \sin \frac{\alpha}{2}+2 \sin \alpha \sin \frac{(2 m+1) \alpha}{2} \sin \frac{\alpha}{2}=0,
$$


or equivalently,

$0=\cos \frac{\alpha}{2} \cos \frac{(2 m+1) \alpha}{2}+\sin \frac{\alpha}{2} \sin \frac{(2 m+1) \alpha}{2}=\cos \left(\frac{\alpha}{2}-\frac{(2 m+1) \alpha}{2}\right)=\cos m \alpha$

Hence

$$
\alpha=\alpha_{k}=\frac{2 k+1}{2 m} \pi
$$

and (??) follows. 